\title{
Atrial fibrillation following off-pump versus on-pump coronary artery bypass grafting: Incidence and risk factors
}

\author{
Łukasz Lewicki ${ }^{1,2}$, Janusz Siebert ${ }^{1,2}$, Jan Rogowski ${ }^{3}$ \\ ${ }^{1}$ University Center for Cardiology, Medical University of Gdansk, Poland \\ ${ }^{2}$ Department of Family Medicine, Medical University of Gdansk, Poland \\ ${ }^{3}$ Department of Cardiac Surgery, Medical University of Gdansk, Poland
}

\begin{abstract}
Background: Postoperative atrial fibrillation $(A F)$ is a common arrhythmia that occurs after coronary artery bypass grafting $(C A B G)$. New surgical techniques, particularly off-pump coronary artery bypass (OPCAB), are thought to be less invasive and results in fewer complications, i.e. AF, but available data are inconsistent. The aim of this study is to present the incidence and risk factors of $A F$ in patients operated on with or without cardiopulmonary bypass.

Methods: We studied 1836 consecutive patients with stable coronary artery disease who were operated on with $(C A B G)$ or without $(O P C A B)$ cardiopulmonary bypass. The patients were monitored using a continuous electrocardiogram monitoring system until the sixth postoperative day.

Results: Atrial fibrillation occurred in $18.3 \%$ and $19.3 \%$ of $C A B G$ and OPCAB patients, respectively $(p=0.3)$. The peak incidence of arrhythmia was observed between the second and third postoperative day in both $C A B G$ and $O P C A B$ patients (36\% and $41 \%$, respectively). $P a-$ tient's age and history of hypertension were significant predictors of postoperative $A F(O R$ 1.38, 95\% CI 1.01-1.76, $p=0.0002$; and OR 1.38, 95\% CI 1.01-1.76, $p=0.008$, respectively). Patients who developed $A F$ vs. without $A F$ had significantly higher rates of complications such as death (3.1\% vs. $1.2 \%, p=0.01)$, reoperation ( $5.2 \%$ vs. $2.8 \%, p=0.02)$, and the need to utilize intra-aortic balloon pump (IABP) (6.8\% vs. 3.4\%, $p=0.002)$. Use of IABP and reoperation were significant perioperative predictors of the arrhythmia (OR 2.1, 95\% CI 1.27-3.4, $p=0.003$; and OR 1.9, 95\% CI 1.09-3.30, $p=0.02$, respectively). AF was also associated with a prolonged stay in an intensive care unit (72.5 \pm 78.8 for patients with $A F$ vs. $34.6 \pm 25.2$ for patients with sinus rhythm, $p=0.000001$ ).
\end{abstract}

Conclusions: In patients undergoing $C A B G$, postoperative $A F$ is a common arrhythmia independent of the type of surgical procedure. (Cardiol J 2016; 23, 5: 518-523)

Key words: atrial fibrillation, cardiopulmonary bypass, coronary artery bypass grafting, off-pump coronary artery bypass

Address for correspondence: Łukasz Lewicki, MD, PhD, Department of Family Medicine, Medical University of Gdansk, ul. Dębinki 2, 80-210 Gdańsk, Poland, tel: +48 5834915 75, +48 501702 885, fax: 48583491576 ,

e-mail: luklewicki@gmail.com

Received: 27.04.2016 Accepted: 25.05.2016 


\section{Introduction}

Postoperative atrial fibrillation (AF) is the most common arrhythmia complicating coronary artery bypass grafting (CABG). It is reported in 15-33\% of patients undergoing coronary bypass surgery [1-5]. A comprehensive review of data concerning postoperative $\mathrm{AF}$ was published by Banach et al. [6].

Although in some studies it has been considered a benign and self-limiting complication, some data suggest serious morbidity and an increase in outcomes in patients who developed AF postoperatively $[7,8]$. In a large study, Mariscalco et al. [9] found that long-term mortality rates were significantly higher in patients with postoperative AF. The arrhythmia significantly increases the risk of stroke [10]. A hypothesis that off-pump coronary artery bypass (OPCAB) is associated with less frequent postoperative $\mathrm{AF}$ and improved outcomes have been tested in several studies but available data are inconsistent [11-15].

The aim of the study was to evaluate the incidence and predictors of AF in patients operated with or without cardiopulmonary bypass.

\section{Methods}

We studied 1836 consecutive patients with documented stable coronary artery disease (CAD) operated in the Department of Cardiac Surgery, Medical University of Gdansk, over a 63-month period. The study protocol was approved by the local bioethics committee. The inclusion criteria were: (1) angiographically documented stable CAD referred for cardiac surgery; and (2) sinus rhythm (SR) in a standard 12-lead electrocardiogram (ECG) performed before the surgery upon hospital admission.

The exclusion criteria included persistent/permanent $\mathrm{AF}$ or a history of paroxysmal $\mathrm{AF}$ before the surgery, permanent cardiac pacemaker implanted before the surgery, concomitant valvular heart disease, other surgical procedure than isolated $\mathrm{CABG}$ or $\mathrm{OPCAB}$, and an acute coronary syndrome.

Patients were divided into two groups depending on whether they were operated with $\mathrm{CABG}$ or $\mathrm{OPCAB}$ technique. The decision for using or not using OPCAB technique was made by a surgeon performing the procedure and was related to specific clinical and anatomical conditions.

The same surgical staff presenting a comparable level of experience performed both $\mathrm{CABG}$ and OPCAB procedures.
All patients were managed using a similar perioperative protocol. Beta-blockers were administered before the surgery in the majority of patients and continued immediately after the surgical procedure. No other antiarrhythmic protocol was used in the prevention of postoperative AF.

A median sternotomy was performed in both CABG and OPCAB groups. A modified Saint Thomas cardioplegic solution was used in the CABG group.

\section{Arrhythmia analysis}

Atrial fibrillation was assessed by the use of continuous ECG monitoring system (Hewlett Packard, USA) with the possibility of rhythm disturbance analysis during the patient stay in an intensive care unit (ICU). Subsequently, during the first $24 \mathrm{~h}$ after leaving the ICU, each patient was monitored using the Space Lab system (Space Lab, USA). A standard 12-lead ECG was performed once a day and in the cases of clinical manifestations of an arrhythmia. The analysis took into account all AF events lasting longer than $10 \mathrm{~min}$ or requiring medical treatment due to clinical instability. The period of analysis was restricted to 6 postoperative days.

\section{Statistical analysis}

Results are expressed as mean values and standard deviation. The EuroSCORE data are median values and ranges and were compared using the Kruskal-Wallis test. Categorical variables were evaluated with the $\chi^{2}$ test, normally distributed continuous variables with the Student $t$ test, and non-normally distributed continuous variables with the Mann-Whitney U test. Odds ratios (OR) were calculated using logistic regression model. $\mathrm{P}<0.05$ was considered statistically significant. Statistical analyses were performed using the Statistica 10.0 (Statsoft) software.

\section{Results}

The mean patient age was 61.6 years and men comprised the majority (75.5\%) of the study population. More than half of patients had a history of hypertension. In the study group, 1051 (57.2\%) patients were operated with cardiopulmonary bypass (CPB) and $785(42.8 \%)$ patients were operated without CPB.

Preoperative data of 1836 patients were divided into two groups depending on the type of the procedure are shown in Table 1.

The OPCAB group had a higher proportion of women $(27.4 \%$ vs. $22.3 \%, \mathrm{p}=0.008)$, patients with 
Table 1. Comparison of perioperative data of 1836 patients according to the type of the procedure.

\begin{tabular}{|c|c|c|c|}
\hline & CABG $(n=1051)$ & OРCAB $(n=785)$ & $\mathbf{P}$ \\
\hline Age [years] & $60.8 \pm 8.9$ & $62.6 \pm 10.2$ & 0.2 \\
\hline Gender: & & & 0.008 \\
\hline Male & $817(77.7 \%)$ & $234(22.3 \%)$ & \\
\hline Female & $570(72.6 \%)$ & $215(27.4 \%)$ & \\
\hline Body mass index $\left[\mathrm{kg} / \mathrm{m}^{2}\right]$ & $27.5 \pm 4.03$ & $27.2 \pm 3.7$ & 0.6 \\
\hline Previous myocardial infarction & $536(50.9 \%)$ & $478(60.9 \%)$ & 0.00002 \\
\hline Hypertension & $620(58.9 \%)$ & $541(68.9 \%)$ & 0.00003 \\
\hline Diabetes & $216(20.5 \%)$ & $184(23.4 \%)$ & 0.2 \\
\hline Chronic obstructive pulmonary disease & $25(2.4 \%)$ & $28(3.5 \%)$ & 0.1 \\
\hline Peripheral arterial disease & $86(8.2 \%)$ & $112(14.2 \%)$ & 0.00004 \\
\hline Neurologic deficit & $72(6.8 \%)$ & $32(4.1 \%)$ & 0.08 \\
\hline Beta-blocker & $845(80.6 \%)$ & $674(85.8 \%)$ & 0.3 \\
\hline Angiotensin converting enzyme inhibitor & $662(62.9 \%)$ & $511(65.1 \%)$ & 0.6 \\
\hline Statin & $736(70.0 \%)$ & $573(72.9 \%)$ & 0.8 \\
\hline EuroSCORE [median (min; max)] & $3(0 ; 11)$ & $3(0 ; 10)$ & 0.2 \\
\hline Left ventricular ejection fraction [\%] & $53.6 \pm 11.7$ & $53.4 \pm 11.1$ & 0.7 \\
\hline Number of distal anastomoses & $3.4 \pm 0.7$ & $2.5 \pm 0.8$ & 0.001 \\
\hline Creatine kinase isoenzyme $\mathrm{MB}[\mathrm{ng} / \mathrm{mL}]$ & $57.2 \pm 13.0$ & $7.6 \pm 1.3$ & 0.003 \\
\hline Drainage on $1^{\text {st }}$ day $[\mathrm{mL}]$ & $551.5 \pm 305.6$ & $668.6 \pm 303.3$ & 0.3 \\
\hline Time in intensive care unit $[\mathrm{h}]$ & $26.8 \pm 14.2$ & $27.3 \pm 17.3$ & 0.9 \\
\hline
\end{tabular}

Data are expressed as mean \pm standard deviation or number (percentage). The EuroSCORE data are expressed as median and minimum and maximum; CABG — coronary artery bypass grafting; OPCAB - off-pump coronary artery bypass

a history of arterial hypertension ( $68.9 \%$ vs. $58.9 \%$, $\mathrm{p}=0.00003)$ and peripheral arterial disease $(14.2 \%$ vs. $8.2 \%, \mathrm{p}=0.00004)$. Patients in the $\mathrm{CABG}$ group were characterized by a higher number of distal anastomoses performed ( $3.4 \pm 0.7$ vs. $2.5 \pm$ $\pm 0.8, \mathrm{p}=0.001)$ and higher postoperative levels of creatine kinase isoenzyme $\mathrm{MB}(57.2 \pm 13 \mathrm{vs}$. $7.6 \pm 1.3, \mathrm{p}=0.003)$ than patients in the OPCAB group All these data are summarized in Table 1.

The mean aortic cross-clamp time in the CABG group was $44.1 \pm 13.9 \mathrm{~min}$, and the mean CPB time was $86.6 \pm 51.1 \mathrm{~min}$.

The length of postoperative stay in the ICU did not differ between the two groups (Table 1).

Postoperatively, AF was observed in 344 (18.7\%) patients. The AF incidence was $18.3 \%$ in the CABG group and $19.3 \%$ in the OPCAB group (OR 0.88, 95\% confidence interval [CI] 0.69-1.12, $\mathrm{p}=0.3)$.

The peak incidence of $\mathrm{AF}$ was observed on the second and third postoperative daysimilarly in both CABG and OPCAB patients (Fig. 1).

Patients who developed postoperative AF were significantly older, more often hypertensive,

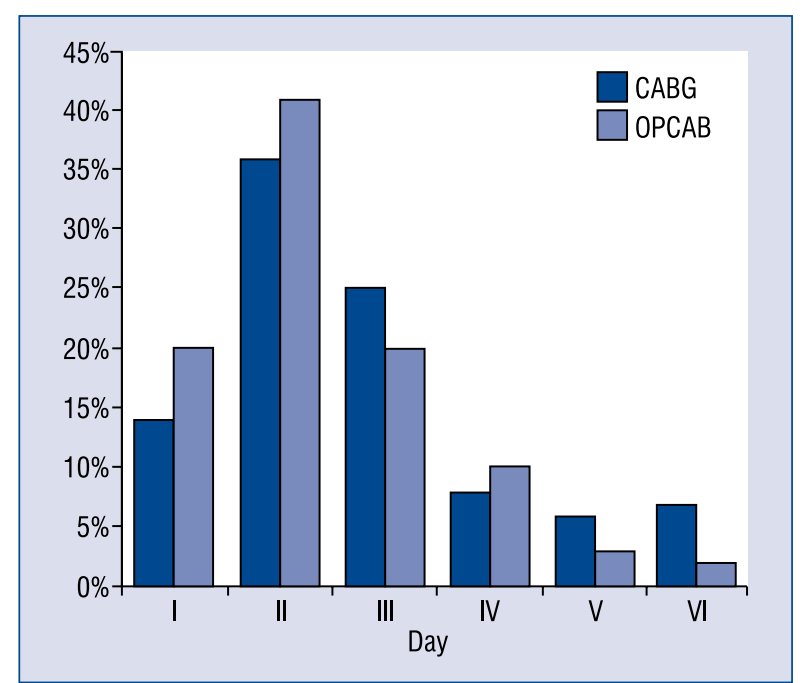

Figure 1. Daily distribution of atrial fibrillation incidence in 344 patients operated with or without cardiopulmonary bypass; CABG - coronary artery bypass grafting; OPCAB — off-pump coronary artery bypass.

and had higher EuroSCORE values than those who remained in SR (Table 2). Hypertension and age 
Table 2. Clinical data of 1836 patients with or without postoperative atrial fibrillation (AF).

\begin{tabular}{lccc}
\hline & AF $(\mathbf{n}=344)$ & SR $(\mathbf{n}=1492)$ & P \\
\hline Age $[$ years] & $63.7 \pm 8.5$ & $60.5 \pm 9.4$ & 0.0002 \\
Body mass index $\left[\mathrm{kg} / \mathrm{m}^{2}\right]$ & $27.3 \pm 4.1$ & $27.4 \pm 3.6$ & 0.9 \\
Gender: & & & 0.1 \\
$\quad$ Male & $249(72.4 \%)$ & $1138(76.3 \%)$ & $353(23.6 \%)$ \\
$\quad$ Female & $94(27.3 \%)$ & $810(54.3 \%)$ & 0.07 \\
Previous myocardial infarction & $204(59.3 \%)$ & $924(61.9 \%)$ & 0.008 \\
Hypertension & $238(69.2 \%)$ & $317(21.2 \%)$ & 0.3 \\
Diabetes & $91(26.4 \%)$ & $38(2.5 \%)$ & 0.08 \\
Chronic obstructive pulmonary disease & $14(4.1 \%)$ & $167(11.2 \%)$ & 0.3 \\
Peripheral arterial disease & $32(9.3 \%)$ & $1253(83.9 \%)$ & 0.7 \\
Beta-blocker & $281(81.6 \%)$ & $1018(68.2 \%)$ & 0.1 \\
Angiotensin converting enzyme inhibitor & $208(60.5 \%)$ & $1137(76.2 \%)$ & 0.7 \\
Statin & $250(72.6 \%)$ & $53.8 \pm 12.4$ & 0.3 \\
Left ventricular ejection fraction $[\%]$ & $52.5 \pm 13.4$ & $2.91 \pm 2.2$ & 0.02 \\
EuroScoRE & $3.4 \pm 2.5$ & & \\
\hline
\end{tabular}

Data are expressed as mean \pm standard deviation or number (percentage); SR - sinus rhythm

were independent risk factors for postoperative AF (OR 1.38, 95\% CI 1.01-1.76, p = 0.008; and OR $1.38,95 \%$ CI $1.01-1.76, \mathrm{p}=0.0002$ ), With each decade over 40 years, a $10 \%$ increase in $\mathrm{AF}$ incidence was observed (Fig. 2).

The length of ICU stay was significantly longer in patients who developed postoperative $\mathrm{AF}$ than in those with SR (Table 3). This difference remained significant even after patients with complications other than AF were excluded from the analysis $(47.8 \pm 33.1$ vs. $32.1 \pm 19.3 \mathrm{~h}$ in patients with $\mathrm{AF}$ and SR, respectively).

Patients with AF had significantly higher rates of complications such as death, reoperation and the need to use an intra-aortic balloon pump (IABP) (Table 4).

Overall in-hospital mortality was $1.63 \%$ (30 of 1836 patients). In a multivariate regression model, postoperative AF was not associated with mortality. Independent risk factors for mortality were

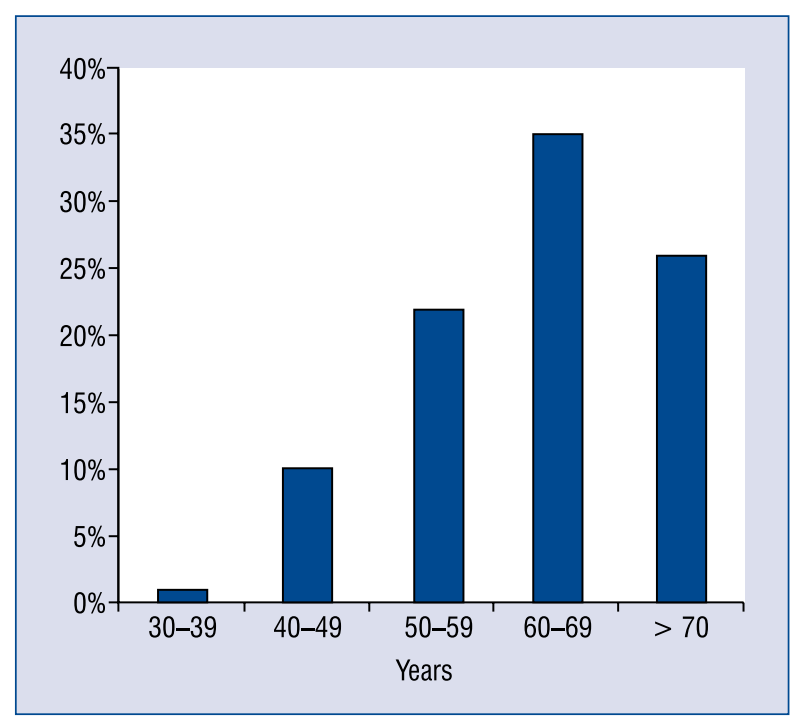

Figure 2. Atrial fibrillation incidence in relation to the age of operated patients.

Table 3. Perioperative data of 1836 patients with or without postoperative atrial fibrillation (AF).

\begin{tabular}{lccc}
\hline & AF $(\mathbf{n}=344)$ & SR $(\mathbf{n}=1492)$ & P \\
\hline Time in intensive care unit $[\mathrm{h}]$ & $72.5 \pm 78.8$ & $34.6 \pm 25.2$ & 0.000001 \\
Creatine kinase isoenzyme $\mathrm{MB}[\mathrm{ng} / \mathrm{mL}]$ & $11.1 \pm 5.7$ & $41.9 \pm 10.3$ & 0.5 \\
Drainage on $1^{\text {st }}$ day $[\mathrm{mL}]$ & $611.6 \pm 345.9$ & $589.7 \pm 283.5$ & 0.8 \\
Number of distal anastomoses & $3.1 \pm 0.9$ & $3 \pm 0.9$ & 0.3 \\
\hline
\end{tabular}

Data are expressed as mean \pm standard deviation; SR - sinus rhythm 
Table 4. Perioperative complications in 1836 patients with or without postoperative atrial fibrillation (AF).

\begin{tabular}{lccc}
\hline & AF $(\mathbf{n}=344)$ & SR $(\mathbf{n}=1492)$ & $P$ \\
\hline Death & $12(3.1 \%)$ & $18(1.2 \%)$ & 0.01 \\
Reoperation & $20(5.2 \%)$ & $41(2.8 \%)$ & 0.02 \\
Intra-aortic balloon pump & $26(6.8 \%)$ & $49(3.4 \%)$ & 0.002 \\
\hline
\end{tabular}

Data are expressed as number (percentage); SR - sinus rhythm

Table 5. Predictors of postoperative atrial fibrillation in a logistic regression model.

\begin{tabular}{lcc}
\hline & $\begin{array}{c}\text { Odds ratio } \\
(\mathrm{CI})\end{array}$ & P \\
\hline IABP & $2.1(1.27-3.4)$ & 0.003 \\
Reoperation & $1.9(1.09-3.3)$ & 0.02 \\
Successful resuscitation & $1.2(0.5-1.5)$ & 0.2 \\
Respiratory distress & $1.4(0.3-2)$ & 0.4 \\
Psychosis & $0.9(0.6-1.3)$ & 0.6 \\
Pneumothorax & $0.9(0.1-1.2)$ & 0.8 \\
Acute renal failure & $1.3(0.08-1.5)$ & 0.6 \\
Need for epicardial pacing & $0.8(0.3-1.3)$ & 0.8 \\
Pleural effusion & $1.2(0.09-1.8)$ & 0.7 \\
Infection & $1.4(0.6-1.9)$ & 0.4 \\
\hline
\end{tabular}

$\mathrm{Cl}$ - confidence interval; IABP — intra-aortic balloon pump

reoperation $(\mathrm{p}=0.002)$, IABP use $(\mathrm{p}=0.009)$, and peripheral arterial disease $(\mathrm{p}=0.01)$.

In a logistic regression model, reoperation and the need for perioperative IABP were significant predictors of postoperative AF (Table 5).

None of the following complications: successful resuscitation, respiratory distress, psychosis, pneumothorax, acute renal failure, pleural effusion infection were indicated to have independent association with AF (Table 5).

In the study population, the predominant strategy of SR restoration was administration of amiodarone (77.6\%). Electrical cardioversion was performed in only $7.8 \%$ of patients. Among 314 patients who developed $\mathrm{AF}$ and survived the surgery, SR was restored in $247(78.6 \%)$, and the remaining $67(21.3 \%)$ were discharged to stepdown units with AF.

\section{Discussion}

The question of whether postoperative AF is less frequent after off-pump cardiac surgery is still unresolved. One recently published meta-analysis of 43 randomized clinical trials has shown no sig- nificant difference between $\mathrm{CABG}$ and $\mathrm{OPCAB}$ in the incidence of stroke and perioperative myocardial infarction but the incidence of postoperative $\mathrm{AF}$ was significantly reduced in the OPCAB group [16]. Another large multicentre trial that compared the safety of CABG and OPCAB in octogenarians showed a reduced risk of $\mathrm{AF}$ in patients operated without CPB [17].

The incidence of postoperative AF in our study was similar in both CABG and OPCAB groups and the difference was not significant. This is consistent with our previously published findings based on a smaller number of patients [18]. Recently, Bohatch Junior et al. [19] demonstrated no significant difference in the incidence of postoperative $\mathrm{AF}$ between on-pump and off-pump patients.

Most AF episodes were noted on the second and third postoperative day. This trend was similar in CABG and OPCAB patients. The observed peak $\mathrm{AF}$ incidence corresponds to some literature data suggesting that in patients after $\mathrm{CABG}$, the highest activation of the complement system also occurs between the second and third postoperative day [20]. The hypothesis that eliminating OPCAB leads to a reduced postoperative inflammatory response was not confirmed by Czerny at al. [21] which showed no significant difference in postoperative levels of interleukin (IL)-6 and IL-10 between patients operated with or without CPB. Another study showed a significant increase in IL-6, IL-8, and C-reactive protein levels in patients after OPCAG, although only IL-6 correlated significantly with postoperative AF [22]. In the present study it was duly noted that daily distribution of postoperative $\mathrm{AF}$ incidence did not differ between CABG and OPCAB patients.

In addition, a relation has been suggested between increased oxidative stress and postoperative AF [23]. More recently, Dehghani et al. [24] noted a role of oral vitamin $\mathrm{C}$ in the prevention of $\mathrm{AF}$ after CABG. These data support the inflammatory/ /oxidative stress pathophysiology of postoperative arrhythmia.

The present study found that a need for reoperation or perioperative IABP use were independ- 
ent risk factors for the development of postoperative AF. This is consistent with the data reported by Mariscalco et al. [25] who found that preoperative IABP was an independent predictor of AF.

Although AF was not an independent risk factor for in-hospital mortality, it could be a marker of a complicated postoperative course.

Postoperative AF incidence was a significant risk factor of prolonged ICU stay, even after adjustment for the presence of other complications. A probable explanation may be the fact that tachycardia and loss of coordinated atrial function during $\mathrm{AF}$ may enhance hemodynamic instability and compromise patient's postoperative clinical condition when compared to those who remain in SR.

In this study, age was a strong risk factor for the development of postoperative AF. This corresponds to other data [1-3]. More recently, VilesGonzalez et al. [26] have duly noted an advanced age was the strongest risk factor for $\mathrm{AF}$ after mitral valve repair.

A possible limitation of our study was a higher number of patients with hypertension in the $\mathrm{OPCAB}$ group. As hypertension has been indicated as an independent risk factor for postoperative AF, it could have influenced the results.

\section{Conclusions}

Our study provides data from a large singlecentre group of consecutive patients operated with or without $\mathrm{CPB}$ suggesting that postoperative $\mathrm{AF}$ is a frequent arrhythmia independent of the type of surgical procedure and it occurs more frequently in patients with an increased risk of serious perioperative complications.

\section{Conflict of interest: None declared}

\section{References}

1. Aranki SF, Shaw DP, Adams DH et al. Predictors of atrial fibrillation after coronary artery surgery. Current trends and impact on the hospital resources. Circulation, 1996; 94: 390-397.

2. Creswell LL, Schuessler RB, Rosenbloom M, Cox JL. Hazards of postoperative atrial arrhythmias. Ann Thorac Surg, 1993; 56: 539-549.

3. Almassi GH, Schowalter T, Nikolosi AC et al. Atrial fibrillation after cardiac surgery: A major morbid event? Ann Surg, 1997; 226: 501-513.

4. Maisel W, Rawn J, Stevenson W. Atrial fibrillation after cardiac surgery. Ann Intern Med, 2001; 135: 1061-1073.

5. Mathew JP, Fontes ML, Tudor IC et al. A multicenter risk index for atrial fibrillation after cardiac surgery. JAMA, 2004; 291: 1720-1729.

6. Banach M, Kourliouros A, Reinhart KM et al. Postoperative atrial fibrillation: What do we really know? Curr Vasc Pharmacol, 2010; 8: 553-572.
7. Mariscalco G, Engstrom KG. Postoperative atrial fibrillation is associated with late mortality after coronary surgery, but not after valvular surgery. Ann Thorac Surg, 2009; 88: 1871-1876.

8. Villareal RP, Hariharan R, Liu BC et al. Postoperative atrial fibrillation and mortality after coronary artery bypass surgery. J Am Coll Cardiol, 2004; 43: 742-748.

9. Mariscalco G, Klersy C, Zanobini M et al. Atrial fibrillation after isolated coronary surgery affects late survival. Circulation, 2008; 118: 1612-1618.

10. Barbieri LR, Sobral ML, Geronimo GM et al. Incidence of stroke and acute renal failure in patients of postoperative atrial fibrillation after myocardial revascularization. Rev Bras Cir Cardiovasc, 2013; 28: 442-448.

11. Boyd WD, Desai ND, Del Rizzo DF, Novick RJ, McKenzie FN, Menkis AH. Off-pump surgery decreases postoperative complications and resource utilization in the elderly. Ann Thorac Surg, 1999; 68: 1490-1493.

12. Ascione R, Caputo M, Calori G, Lloyd CT, Underwood MJ, Angelini GD. Predicors of atrial fibrillation after conventional and beating heart coronary surgery. A prospective, randomized study. Circulation, 2000; 102: 1530-1535.

13. Salamon T, Michler RE, Knott KM, Brown DA. Off-pump coronary artery bypass grafting does not decrease the incidence of atrial fibrillation. Ann Thorac Surg, 2003; 75: 505-507.

14. Singhal P, Mahon B, Riordan J. A prospective observational study to compare conventional coronary artery bypass grafting surgery with off-pump coronary artery bypass grafting on basis of EuroSCORE. J Card Surg, 2010; 25: 495-500.

15. Scherer M, Sirat AS, Dogan S, Aybek T, Moritz A, WimmerGreinecker G. Does totally endoscopic access for off-pump cardiac surgery influence the incidence of postoperative atrial fibrillation in coronary artery bypass grafting? A preliminary report. Cardiovasc Eng, 2006; 6: 118-121.

16. Chen Y, Shu J, Yang W et al. Meta-analysis of randomized trials comparing the effectiveness of on-pump and off-pump coronary artery bypass. Chin Med J, 2012; 125: 338-344.

17. LaPar DJ, Bhamidipati CM, Reece TB, Cleveland JC, Kron IL, Ailawadi G. Is off-pump coronary artery bypass grafting superior to conventional bypass in octogenarians? J Thorac Cardiovasc Surg, 2011; 141: 81-90.

18. Siebert J, Anisimowicz L, Lango R et al. Atrial fibrillation after coronary artery bypass grafting:does the type of procedure influence the early postoperative incidence? Eur J Cardio-thorac Surg, 2001; 19: 455-459.

19. Bohatch Junior MS, Matkovski PD, Di Giovanni FJ, Fenili R, Varella EL, Dietrich A Incidence of postoperative atrial fibrillation in patients undergoing on-pump and off-pump coronary artery bypass grafting. Braz J Cir Cardiovasc, 2015; 30: 316-324.

20. Bruins P, te Velthuis H, Yazdanbakhsh AP et al. Activation of the complement system during and after cardiopulmonary bypass surgery. Circulation, 1997; 96: 3542-3548.

21. Czerny M, Baumer H, Kilo J et al. Inflammatory response and myocardial injury following coronary artery bypass grafting with or without cardiopulmonary bypass. Eur J Cardio-thorac Surg, 2000; 17: 737-742.

22. Ishida K, Kimura F, Imamaki M et al. Relation of inflammatory cytokines to atrial fibrillation after off-pump coronary artery bypass grafting. Eur J Cardiothorac Surg, 2006; 29: 501-505.

23. Elahi MM, Flatman S, Matata BM. Tracing the origins of postoperative atrial fibrillation: The concept of oxidative stress-mediated myocardial injury phenomenon. Eur J Cardiovasc Prev Rehabil, 2008; 15: 735-741.

24. Dehghani MR, Madjidi N, Rahmani A, Asgari B, Rezaei Y. Effect of oral vitamin $\mathrm{C}$ on atrial fibrillation development after isolated coronary artery bypass grafting surgery: A prospective randomized clinical trial. Cardiol J, 2014; 21: 492-499.

25. Mariscalco G, Biancaari F, Zanobini M et al. Bedside tool for predicting the risk of postoperative atrial fibrillation after cardiac surgery: the POAF score. J Am Heart Assoc, 2014; 3: e000752.

26. Viles-Gonzalez JF, Enriquez AD, Castillo JG et al. Incidence, predictors, and evolution of conduction disorders and atrial arrhythmias after contemporary mitral valve repair. Cardiol J, 2014; 21: 569-575. 